\title{
Equidad en el Acceso a los Servicios de Salud y Equidad en la Financiación de la Atención en Bogotá
}

\author{
Equity in gaining access to health services and equity in funding \\ being attended by them in Bogota
}

\author{
Martha L. Rubio-Mendoza \\ Fondo de Población de las Naciones Unidas UNFPA. rubio@unfpa.org \\ Recibido 8 Enero 2007/Enviado para Modificación 2 Mayo 2008/Aceptado 31 Octubre 2008

\section{RESUMEN}

Objetivo Identificar inequidades en el acceso y financiación de la atención en salud en Bogotá.

Métodos Se efectuó un análisis de la Encuesta de Calidad de Vida y Salud del año 2003 para Bogotá. Se identificaron variables socioeconómicas, de necesidad, acceso a los servicios y financiación. Se realizó análisis descriptivo y bivariado, recodificación de variables y creación de escenarios que combinaban las variables de necesidad, acceso y financiación de la atención.

Resultados El 18,4 \% de population en régimen contributivo y el 26,8 \% del régimen subsidiado no acceden a los servicios de salud. La principal barrera de acceso para la no utilización de los servicios de salud es la falta de dinero, aún en la population afiliada al SGSSS. Las localidades que presentan mayor inequidad en el acceso a los servicios de salud son San Cristóbal, Kennedy Rafael Uribe. A partir de los escenarios construidos se configuraron dos perfiles, el de mayor inequidad, el cual está determinado por pertenecer al quintil 1 y 2, ser hombre, estar entre los 0 y 18 años, estar en el régimen subsidiado o no estar afiliado al SGSSS. Y el segundo que ofrece mejores condiciones de equidad, el cual está relacionado con ser mujer, mayor de 19 años, pertenecer a los quintiles 3, 4 o 5 y estar afiliado al régimen contributivo.

Conclusión Se observa inequidad en el acceso a los servicios de salud en particular en la population más pobre, aún estando afiliados al SGSSS.

Palabras Clave: Equidad en salud, equidad en el acceso, Bogotá (fuente: DeCS, BIREME).

\section{ABSTRACT}

Objective Identifying inequity in gaining access to and financing health service attention in Bogotá.

Methods The 2003 Quality of Life and Health Survey for Bogotá was analysed. Socioeconomic, needs, access to health services and financing them were identified 
as variables. A descriptive and bivariate analysis was done, variables were re-codified and scenarios were created combining variables regarding needs, access and financing attention.

Results $18,4 \%$ of the population in the contributory regimen and $26,8 \%$ of the subsidised regime were not gaining access to health services. The main barrier to gaining access for using health services is the lack of money, even in the population affiliated to the social health security system (SHSS). The localities presenting the greatest inequity in access to health services were San Cristóbal, Kennedy and Rafael Uribe. Two profiles were configured from the scenarios so constructed: that having greatest inequity (determined by belonging to the $1^{\text {st }}$ and $2^{\text {nd }}$ quintiles, being male, being aged 0-18 years old, being in the subsidised regimen or not being affiliated to the SHSS) and that offering the best conditions of equity (related to being female, aged over 19 , belonging to the $3^{\text {rd }}, 4^{\text {th }}$ or $5^{\text {th }}$ quintiles and being affiliated to the contributory regimen).

Conclusion Inequity in gaining access to health services was observed, particularly in the poorest population, even though being affiliated to the SHSS.

Key Words: Equity in health provision, equity in gaining access, Bogotá (source: $\mathrm{MeSH}, \mathrm{NLM})$.

$\mathrm{U}$ na de las principales preocupaciones que en la actualidad ocupa a académicos, administradores, profesionales de la salud entre otros; es la de conocer el impacto que en términos de equidad en el acceso a los servicios de salud y la financiación de la atención ha tenido la implementación de la Ley 100 de 1993 mediante la cual se creó el Sistema General de Seguridad Social en Colombia (1). Las actuales condiciones de deterioro económico y social del país son un elemento estructural que hace distante el cumplimiento de la meta de universalidad en el aseguramiento, de otra parte existen algunas evidencias que sugieren que la población de mayores necesidades en salud y menores ingresos no acceden a los servicios de salud aún estando afiliados al SGSSS (2). Lo cual induce a gastos de bolsillo de esta población como una manera de solventar la necesidad de atención en salud no provista por el sistema.

En el presente estudio se asume el concepto de equidad propuesto por la Sociedad Internacional de Equidad en Salud quién la define como la ausencia de diferencias sistemáticas y potencialmente evitables en aspectos de salud, entre poblaciones o grupos poblacionales definidos, social, económica, demográfica o geográficamente. Partiendo por reconocer la salud como un derecho fundamental.

El presente estudio efectuó una valoración de la equidad en el acceso y la financiación de la atención en salud. Identificando las diferencias consideradas evitables e injustas (3) en Bogotá y 19 localidades. 
El análisis se realizó a partir de tres elementos centrales: a) las necesidades sentidas exploradas a través de enfermedad en los últimos treinta días, percepción del estado de salud y presencia de enfermedades crónicas b) el acceso a los servicios de salud dadas condiciones de enfermedad, para lo cual se partió de las personas que afirmaron tener enfermedad en los últimos treinta días allí se contempló tanto el aseguramiento al SGSSS, como la utilización de los servicios de salud y las barreras de acceso manifiestas c) financiación de la última atención en salud. Que indaga por el uso de recursos de la seguridad social y/ o de bolsillo para cubrir los gastos de la última atención en salud.

\section{METODOLOGÍA}

Este es un estudio descriptivo, transversal, que empleo información de fuente secundaria disponible en la Encuesta Nacional de Calidad de Vida y Salud, del año 2003 realizada por el Departamento Administrativo Nacional de Estadística de Colombia (DANE). El tamaño de muestra para el país fue de 22942 hogares, y para Bogotá fue de 12771 hogares. El error de estimación, fue menor al $5 \%$ para Bogotá.

Se llevo a cabo la revisión de la estructura de las preguntas del capítulo de salud, para identificar las variables de necesidad sentida, acceso y financiación de la atención. A partir del marco de referencia propuesto, se parte por reconocer que en condiciones de equidad dadas unas necesidades en salud, las personas deben tener igual acceso $(4,5)$ y efectuar el pago de acuerdo a su capacidad, y no estar este acceso limitado por su condición de sexo, edad o ingresos (6).

Desde este criterio se revisó la estructura de las preguntas y se identificaron las siguientes categorías de análisis:

Necesidad

Pretende valorar la percepción del estado de salud y las necesidades manifiestas de las personas relacionadas con su estado de salud actual.

Acceso a los servicios de salud Contempla todas las variables que permiten identificar el estado de afiliación, demanda efectiva de servicios y obstáculos frente a la misma. Para valorar el acceso sólo se contemplo a los que respondieron positivamente a la pregunta "si en los últimos 30 días tuvo alguna, enfermedad, accidente, problema odontológico o cualquier otro problema que no haya implicado hospitalización”. Y se asumió como 
acceso a todas aquellas personas que respondieron que "Para tratar este problema de salud que hizo principalmente”... que acudieron a medico, especialista, homeópata, acupunturista, odontólogo, terapista, promotor de salud, enfermera o institución de salud y los que fueron considerados como no acceso son: los que reportaron que acudieron a boticario, farmaceuta, droguista, tegua, empírico, curandero, yerbatero, comadrona, asistió a terapias alternativas, uso remedios caseros, se auto recetó o hizo nada.

Financiación de la atención en salud

Incluye las variables relacionadas con los pagos que las familias realizan por concepto de afiliación al SGSSS y demás gastos de bolsillo referidos al pago de consultas, exámenes de laboratorio y medicamentos entre otros.

No se evaluó la necesidad, ni el acceso de eventos que hayan requerido hospitalización.

Se efectuó una evaluación descriptiva estratificada por sexo, ingreso y edad para algunas variables, para Bogotá y las localidades, se seleccionaron indicadores para comparaciones entre grupos. Se estratificaron algunas variables por sexo, quintil de ingresos y edad, con el objetivo de identificar inequidades en el acceso y la financiación tanto para el conjunto de la ciudad, como para las localidades. A estas estimaciones les realizó pruebas de significancia estadística (7).

Se construyó una ficha técnica por localidad, en la cual se muestran las estimaciones estratificadas por las variables de sexo, edad y quintil de ingresos.

Con el fin de agrupar las inequidades encontradas tanto para el acceso a los servicios de salud como para la financiación de la atención, se construyeron unos escenarios que ordenan las condiciones de acceso y financiación, a manera de una escala ordinal, que partiera desde la situación de menor inequidad a la peor situación o de mayor inequidad.

Para construir estos escenarios se identificó la población que dijo tener algún problema de salud en los últimos treinta días (635 815 personas 9,2 \%), y de estos los que acudieron a los servicios de salud, (465 264; 73 \%), de este subconjunto se estimaron los que habían utilizado como fuente de financiación la seguridad social, cuantos seguridad social más gastos de bolsillo y por último cuantos utilizaron solamente gastos de bolsillo, para su atención. 
Con el procedimiento antes descrito se definieron los siguientes escenarios:

Escenario I: Es aquel en el cual teniendo una necesidad sentida (enfermedad en los últimos treinta días), las personas acudieron a resolver sus problemas en instituciones de salud, y la fuente de financiación empleada para cubrir la atención provino de la seguridad social en salud.

Escenario II: Es aquel en el cual teniendo una necesidad sentida (enfermedad en los últimos treinta días), las personas acudieron a resolver sus problemas en instituciones de salud, y la fuente de financiación empleada para cubrir la atención provino de la seguridad social en salud más gastos de bolsillo.

Escenario III: Es aquel en el cual teniendo una necesidad sentida (enfermedad en los últimos treinta días), las personas acudieron a resolver sus problemas en instituciones de salud, y la fuente de financiación empleada para cubrir la atención fueron gastos de bolsillo.

Escenario IV: Es aquel en el cual teniendo una necesidad sentida (enfermedad en los últimos treinta días), las personas no acudieron a resolver sus problemas en instituciones de salud.

Posterior a la construcción de estos escenarios, se estableció a través de un modelo de análisis de correspondencia la relación que las variables quintil de ingreso, edad, sexo, y afiliación al SGSSS, tenían con los escenarios (I, II, III y IV), ubicando en un plano cartesiano la distancia de las variables, con respecto a los ejes del modelo, identificando la cercanía del grupo de variables con los escenarios.

Se construyeron modelos explicativos, con el fin de establecer cuál es la relación que existe entre los diferentes escenarios y las variables de interés en el análisis de equidad: sexo, edad, quintil de ingreso y régimen de afiliación se hizo un análisis de correspondencia, en el cual se gráfica en un plano cartesiano el conjunto de variables que dada su proximidad corresponden a un conjunto de condiciones que se acercan o alejan de los diferentes escenarios.

Para el procesamiento y análisis estadístico de los datos se empleo los programas SPSS versión 10 en español, STATA versión 8.0 y SAS versión 8.0. 


\section{RESULTADOS}

Necesidad Sentida

Del total de la población de la ciudad el 9,2 \% (635 815), dijo tener algún problema de salud en los últimos 30 días, de estos el 73 \% (465 254) acudió a los servicios de salud institucionales y el restante $27 \%$ acudió a otros servicios, utilizo remedios, se auto recetó o no hizo nada.

Para el total de Bogotá el $10 \%$ de las mujeres reportaron un problema de salud mientras que los hombres lo hicieron en un 8,3 \%. (Diferencias estadísticamente significativas). Se corrobora que la necesidad sentida es mayor en las mujeres que en los hombres.

Del total de las 19 localidades, las de Kennedy y Suba presentan la mayor proporción de necesidad sentida en los quintiles 1,2 y 3 de la población.

Se observa que en la medida que aumenta el quintil de ingreso la percepción en el estado de salud mejora, el 61 \% de las personas que dijeron percibir como muy bueno su estado de salud se encontraban en los quintiles 4 y 5 , mientras que el $48 \%$ de los que dijeron percibir su salud como mala, se encontraban en los quintiles de ingreso 1 y 2.

Acceso a los servicios de Salud

Para el total de Bogotá, hay mayor acceso en las mujeres, con un 74,7 \% siendo la diferencia estadísticamente significativa, que en los hombres $71,1 \%$. En el quintil 1 y 2 se observa la mayor proporción de población que no accede a los servicios $39 \%$ y $31 \%$ respectivamente y esta sigue un comportamiento inversamente proporcional en la medida que el quintil de ingreso aumenta el acceso se mejora.

Las razones por las cuales las personas estando enfermas, no acudieron a los servicios de salud, se organizaron en dos categorías, aquellas relacionadas con la condición de la persona que no implica necesariamente dificultades en la organización de los servicios para garantizar el acceso a los mismos, el 49,3 \%, argumentó este tipo de razones y las que evidencian barreras de acceso en los servicios corresponde al 50,7 \% de la población que, estando enferma, no accedió a los servicios de salud. Se observa que las personas ubicadas en los quintiles de ingreso uno y dos acuden en mayor proporción al boticario o droguista, mientras que los que más acuden a los médicos son las personas de los quintiles tres, cuatro y cinco. 
Igual que para el total de la ciudad en el quintil uno y dos de las 19 localidades analizadas se ubican la mayor proporción de población que no accede a los servicios de salud, en el quintil uno se encuentra el menor acceso en las localidades de Engativá (53,3 \%), Kennedy (49,4 \%) y Rafael Uribe (46,4 \%), Mártires (58,9 \%) San Cristóbal (45,1%) y Kennedy (37,1 \%).

El grupo de edad que menos accedió a los servicios de salud es el de 19 a 45 años (45,7 \%), seguido por los de 46 a 60 años (15,2 \%), llama la atención que el menor acceso se concentra en la población joven, lo cual puede estar explicado por la no vinculación laboral formal o la no cobertura de los beneficios de la seguridad social.

La principal razón por la cual no solicitó o no recibió atención médica es porque el caso era leve 43,9 \%, seguido de falta de dinero 31,1 \% y mal servicio o distancia en el tiempo de la cita 6,6 \%. El 53 \% de las personas que dijeron no asistir al médico por falta de dinero eran mujeres y el $46 \%$ hombres. El 44,2 \% de los que no asistieron por falta de dinero se encontraban en edades entre los 19 y 45 años. El 46,8 \% de las personas que dijeron no asistir al médico por falta de dinero se ubicaban en el quintil uno mientras que en el quintil 5 era del 4,6\%. La falta de dinero tanto en el régimen contributivo como en el subsidiado fue la tercera y segunda causa respectivamente por la cual las personas no asistieron al médico 9,01 \% y 29,7 \% (Ver Figura 1).

\section{Afiliación al SGSSS}

En Bogotá D.C., el 63 \% de la población se encuentra en el régimen contributivo, el $14,5 \%$ en el subsidiado y $22,4 \%$ no se encuentra afiliado al SGSSS. La afiliación es mayor para las mujeres tanto en el régimen contributivo como en el subsidiado (63,8 \% y $15 \%$ ) respectivamente, los hombres se encuentran en una mayor proporción en la categoría de no afiliados 23,8 \%.

Frente a la distribución por quintil de ingresos se observa que en el régimen contributivo en la medida que aumenta el quintil aumenta la proporción de afiliación, para el quintil 1 esta proporción es del $25 \%$ y para el quintil 5, del 90,2 \%, a su vez en el quintil uno y dos se encuentra la mayor proporción de población subsidiada, y de población no afiliada al SGSSS (35,9 \% y 28,7 \% respectivamente).

Los grupos de edad que menor cobertura de afiliación tienen al SGSSS son los de 19 a 45 años (25,8 \%) y los menores de 5 años (57,1 \%). 
Figura 1. Distribución (\%) de razones de no utilización de servicios de salud,

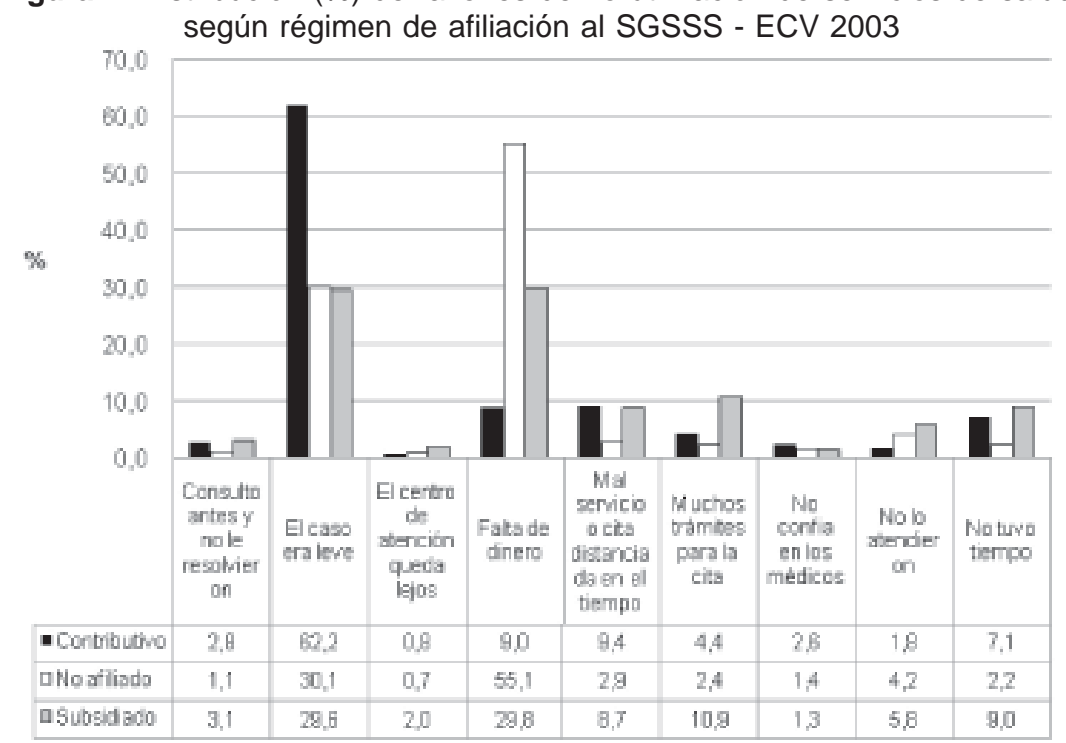

En la Figura 2 se presentan las tres primeras proporciones de afiliación por régimen de afiliación al SGSSS por localidades.

Figura 2. Afiliación al SGSSS en Bogotá

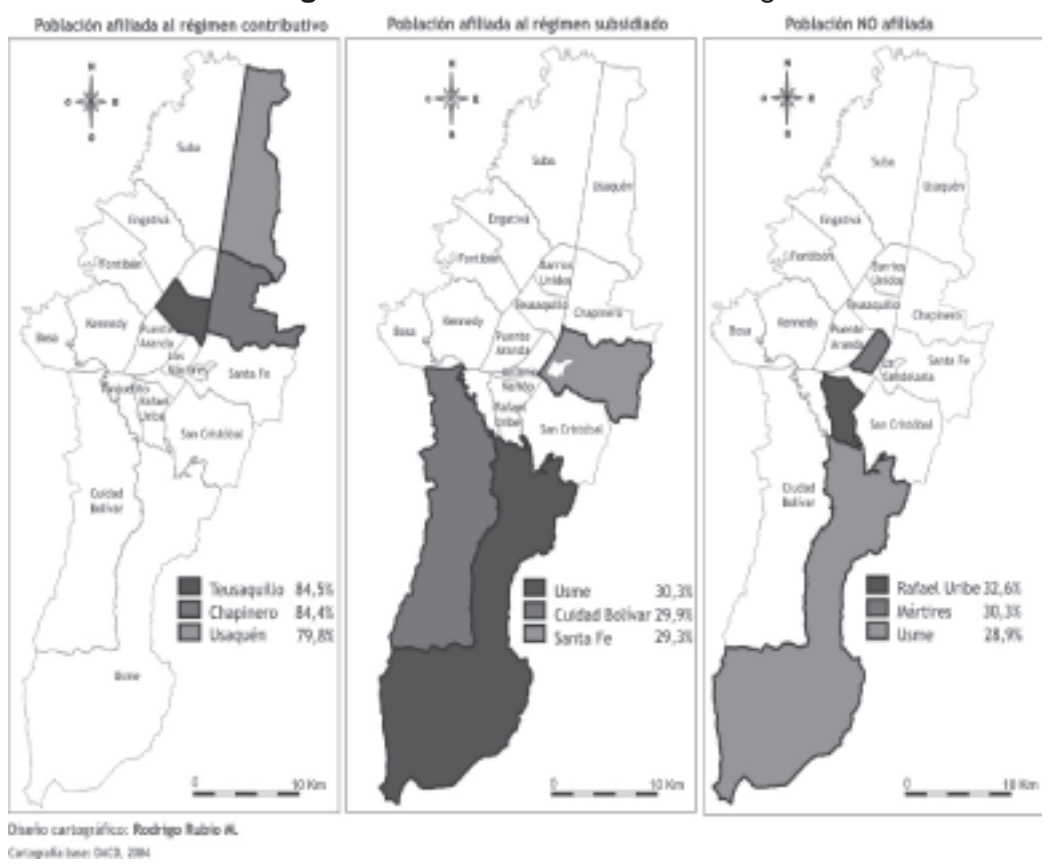


La población subsidiada del quintil uno, de las localidades no supera el 55 \% de la afiliación, en orden descendente Santa Fe, Candelaria y Usaquén (55,3 $\%, 54,8 \%, 50,5 \%$ ), son las que mayor proporción de población afiliada al régimen subsidiado tienen en este quintil; si se compara con los pobres por línea de pobreza de estas localidades (8,9) que son: 52,7 \%, 49 \% y 22,3 \%, respectivamente, se observa una cobertura del más del cien por ciento de la población pobre, ubicada en el quintil uno. Sin embargo, es importante señalar que estas localidades no corresponden a las que mayor proporción de pobres tienen en la ciudad, situación que da cuenta de las dificultades de cobertura del SGSSS y de las estrategias de focalización utilizadas en aquellas poblaciones que por su condición social deberían de estar cubiertas en un 100 \% (10).

Financiación de la Atención

El 35 \% de la población utilizo como fuente de financiación para la última atención la seguridad social más recursos propios, el 33,2 \% la seguridad social y el 31,8 \% recursos propios. Los recursos propios fueron utilizados en mayor proporción por la población no afiliada seguida de los subsidiados y la población del régimen contributivo.

La utilización de recursos propios para la financiación de la atención es inversamente proporcional al quintil, en el quintil uno esta proporción fue del 45 $\%$ mientras que para el quintil cinco del $25 \%$, veinte puntos porcentuales de diferencia de gasto de bolsillo relacionado con la atención en salud, presentándose una clara inequidad.

Creación de escenarios de acceso a los servicios de salud y financiación de la atención

A continuación se presentan los hallazgos de los escenarios estratificados por sexo, edad, afiliación al SGSSS, quintil de ingresos y localidad. El 29,3 \% de la población se ubica en el escenario uno, el $31 \%$ en el escenario II y el 12,4 $\%$ en el escenario III y existe una gran proporción de la población, casi la tercera parte que se encuentra en el escenario IV el 27,2 \% es decir de no acceso. Por sexo la mayor diferencia se observa en el escenario IV, que es mayor en los hombres comparativamente con las mujeres 29,3 \% frente a 25,6 $\%$ respectivamente, lo anterior indica que acceden menos a los servicios; sin embargo las mujeres se ubican en mayor proporción en el escenario II, 32,3 \%, es decir utilizan para la atención en salud aparte de la seguridad social más gastos de bolsillo que los hombres. 
Frente al análisis por edad se observa que las personas mayores de 61 años se encuentran en mayor proporción en el escenario I, mientras que la población entre 18 y 45 años, es la que menor acceso tiene a los servicios de salud, escenario IV. Es importante señalar que en el escenario III, el cual implica acceso a través de la fuente de recursos propios, afecta más a los menores de cinco años (14,3 \%) y a la población de 19 a 45 años 13,9 \%. Frente al escenario II, para todas las edades el comportamiento es similar estando por encima del $27 \%$.

Con respecto a la ubicación de los escenarios por régimen de afiliación al SGSSS, se encuentra que el escenario de mayor equidad, está en el régimen subsidiado 43,4\%, el escenario II, que implica gastos de bolsillo para financiar la atención predomina en el régimen contributivo 40,5 \%, el escenario III y IV naturalmente se observa en mayor proporción en la población no afiliada al SGSSS, 33,1 \% y 52,9 \%. Sin embargo, es de destacar que en el escenario IV, en el régimen contributivo y subsidiado se observa una población considerable, 18,6 \% y 27,4 \% respectivamente, lo cual indica no acceso aún estando afiliados al SGSSS.

En la medida que aumenta el quintil de ingresos la proporción de población que se ubica en el escenario IV se reduce, así por ejemplo el quintil uno tiene un no acceso del 40,2 \% es decir el doble de lo observado en el quintil cinco que es del 20,6 \%. Frente al escenario de máxima equidad el I, el comportamiento es directamente proporcional, es decir a medida que aumenta el quintil de ingresos la proporción de población aumenta, el quintil 1 tiene una proporción de 26,7 \%, mientras que el cinco 35,7 \%.

$\mathrm{Al}$ realizar el análisis de los escenarios por localidad se encuentra que en Chapinero, Santa Fe y Bosa, se halla la mayor proporción de personas en el escenario I, en el escenario II Antonio Nariño, Usaquen y Kennedy, en el III se ubican en orden descendente Teusaquillo, Chapinero y Barrios Unidos, y en la condición de no acceso San Cristóbal, Kennedy y Usme.

Construcción de modelos explicativos

Se construyeron dos modelos, el primero contempla la variable de afiliación y en el segundo se excluyo. Con respecto al primer modelo que se presenta en la Figura 3 se observa que el escenario cuatro es decir el de no acceso está fuertemente relacionado con la condición de pertenecer al quintil uno o dos, ser menor de 18 años, ser hombre, pertenecer al régimen subsidiado o ser no 
afiliado; este conjunto configura las características de no acceso dadas las variables del modelo. El escenario III, está más cerca de la condición de no afiliados y comparte asociación con algunas variables del escenario IV, en especial el ser hombres, pertenecer al quintil II y tener edades entre 0-45 años. Según el modelo se puede estar en el escenario I y II dado que se es mujer, afiliado al régimen contributivo, estar en edades entre los 46 y más años, y pertenecer a los quintiles 3, 4, o 5 . Lo anterior configura dos perfiles de acceso que se ubican en extremos del plano y reflejan las inequidades de la población pobre y joven frente al acceso a los servicios de salud y la financiación en la atención.

Figura 3. Modelo 1: Plano factorial acceso a los servicios de salud y financiación de la atención en función de las variables de equidad. Bogotá D.C. ECV-2003

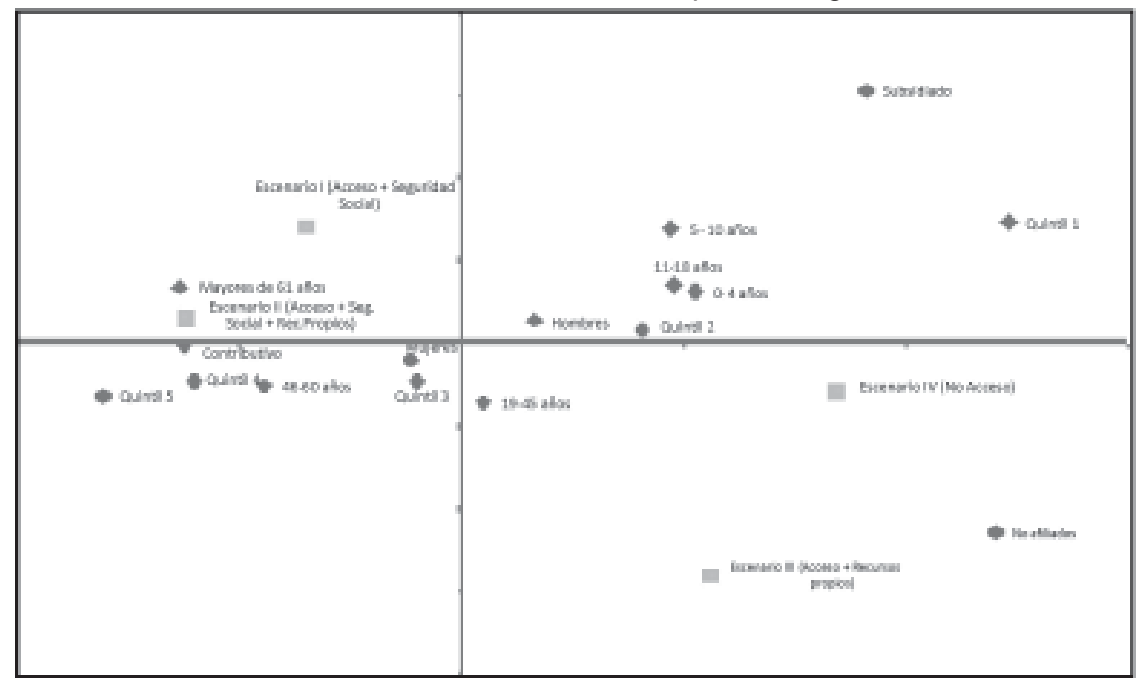

Cuando se excluye del modelo la variable de afiliación al SGSSS, el escenario IV, queda más claramente explicado por la condición de ser menor de 18 años, ser hombre y pertenecer al quintil uno o dos.

\section{DISCUSIÓN}

En la valoración realizada se identificaron inequidades para el total de la población de Bogotá así como para cada una de las localidades, relacionadas con la edad, el quintil de ingresos, el estado de afiliación y el sexo; los métodos empleados permitieron medir estas diferencias, las cuales se señalaron en los resultados y en esta sección se contrastan con algunos estudios. 
Bogotá tiene una de las proporciones más bajas de «enfermedad sentida» para el país. Las mujeres, tal como lo muestran otros estudios tienen una mayor percepción de la necesidad en salud que los hombres. El problema de salud percibido en los últimos treinta días no presentó mayores variaciones en el análisis tanto por quintil de ingresos como por afiliación al SGSSS.

En la medida que aumenta la edad la percepción del estado de salud se deteriora, lo contrario ocurre con el quintil de ingresos, en la medida que aumenta el ingreso la percepción en el estado de salud mejora, situación que refleja las condiciones de bienestar y calidad de vida que tienen las personas de mejor nivel socioeconómico $(11,12)$.

En el acceso se observan inequidades, el 39 \% de la población del quintil uno no accede a los servicios de salud, seguido de la población del quintil 2, lo cual coincide con el hallazgo de Céspedes y cols,(13) que al comparar la utilización de servicios entre 1993 y 1997 por cuartil de ingreso reporto un incremento proporcional por cuartil. Es decir para el año 1993 en el cuartil 1 el acceso era del $5 \%$ mientras que en el cuatro era del 9,9\%; para el año 1997 esta brecha se aumento a más del doble, la utilización en el cuartil 1 para 1997 fue 7,4 \%, mientras que para el cuartil 4 fue de 18,8 \%. Sin embargo en el análisis y discusión de los resultados no se mencionó este hallazgo como una inequidad. Las localidades que reportaron mayores proporciones de no acceso y que podría considerarse críticas son San Cristóbal y Kennedy.

La falta de dinero fue la segunda razón por la cual la población no utilizo los servicios de salud de salud (inclusive los afiliados al régimen contributivo y al subsidiado). Los más afectados: la población del quintil 1, no afiliados al SGSSS, las mujeres, y las personas entre 19 y 45 años. Sin embargo esta razón de no -asistencia fue prevalente tanto en el régimen contributivo como en el subsidiado. Similares hallazgos se observan en el estudio de OPS (14) que concluyó que la falta de dinero es la principal razón de la no -utilización de servicios de salud, indicando que la no atención esta estrechamente relacionado con factores económicos. Y la falta de dinero como razón para la no utilización de servicios de salud entre los afiliados al SGSSS puede estar indicando el papel limitante de la cuota moderadora y del copago, elementos de la falta de financiación del sistema.

Los niños menores de cinco años y los adultos de 19 a 45 años son los que menor afiliación reportan al SGSSS. Según conclusiones del estudio de OPS, 
el porcentaje de afiliación tiende a ser mayor en los grupos de mayor edad, tanto en la zona urbana como rural.

Por regímenes se observa que la afiliación al contributivo se incrementa a medida que incrementa el quintil de ingresos; mientras que en los quintiles $1 \mathrm{y}$ 2 se ubica la mayor proporción de población no asegurada. Lo cual pone en tela de juicio las políticas de focalización del aseguramiento para la población pobre, ya que el 35,7 \% de la población ubicada en el quintil 1 y el 28,7 \% del quintil 2, no esta cubierta por el sistema pese a su condición de pobres. Lo cual evidencia la inequidad del SGSSS. Por el contrario Céspedes y cols, encontraron que entre 1993 y 1997 el aseguramiento se incremento en forma proporcional en todos los déciles de ingreso, siendo más acentuado el crecimiento en el décil uno, en donde la cobertura paso de 3,1 \% en 1993 a 43,7 \%, como se ha planteado en muchas discusiones es explicable este incremento vertiginoso del aseguramiento si se tiene en cuenta que en el año 1993 aún no se había implementado la Ley 100.

Respecto a las fuentes de financiación utilizadas por las personas para el pago de la última atención, se identificaron grandes inequidades; quienes hacen más uso de los recursos de bolsillo son los pobres no afiliados, de los quintiles uno y dos. Las localidades que mayor uso hacen del pago de bolsillo son Tunjuelito, Kennedy, Teusaquillo y San Cristóbal. El pago de bolsillo no es exclusivo de las personas no afiliadas al sistema. El $40 \%$ de personas pertenecientes al régimen contributivo, utilizaron además de la seguridad social gastos de bolsillo para cubrir la atención. En el estudio de Castaño y cols. se encontró que el gasto de bolsillo se redujo significativamente entre 1993 y 1997 para el conjunto de Bogotá; sin embargo este mismo estudio concluía que en general para el país, el quintil 1 no presentaba esta tendencia de descenso.

\section{CONCLUSIONES}

Se evidencia inequidad en el acceso a los servicios de salud, lo cual esta afectando de manera importante a la población más pobre de la ciudad, y la no afiliada al SGSSS principalmente.

Tanto la población afiliada como la no afiliada incurren en gastos de bolsillo para financiar las atenciones en salud, elemento regresivo del sistema que genera inequidades, ya que la población de los quintiles 1 y 2 es la que mayor uso hace esta fuente de financiación. 
La principal barrera de acceso para la no utilización de los servicios de salud la constituye la falta de dinero aún en la población afiliada al SGSSS. Tanto la población afiliada como la no afiliada incurren en gastos de bolsillo para financiar las atenciones en salud.

Se corrobora que aseguramiento no es igual a acceso, ya que una proporción importante de la población afiliada al sistema no accede a los servicios de salud, dada la condición de necesidad expresada en enfermedad en el último mes. El 18,4\% de población en régimen contributivo y el 26,8\% del régimen subsidiado no acceden.

Las localidades que presentan mayor inequidad en el acceso a los servicios de salud son San Cristóbal, Kennedy Rafael Uribe.

Del análisis de los escenarios se concluye que los hombres acceden menos a los servicios de salud, las mujeres acceden más a expensas de la utilización de los gastos de bolsillo combinados con la seguridad social. Los menores de cinco años y la población entre 19 y 45 años es la que más utiliza gastos de bolsillo para garantizar el acceso a los servicios de salud. Entre el $20 \%$ y el 27 \% de la población afiliada no accedió a los servicios de salud. El no acceso en el quintil 1 es dos veces mayor que el del quintil 5.

Se propone la configuración de dos perfiles el primero esta relacionado con condiciones de mayor inequidad es decir estar en el escenario III o IV, el cual esta determinado por pertenecer al quintil 1 y 2 , ser hombre, estar entre los 0 y 18 años, ubicarse en el régimen subsidiado o no estar afiliado al SGSSS. Y el segundo que ofrece mejores condiciones de equidad, es decir pertenecer aI escenario I o II, el cual esta relacionado con ser mujer, mayor de 19 años, pertenecer a los quintiles 3, 4 o 5 y estar afilado al régimen contributivo

\section{REFERENCIAS}

1. Martínez $P$, Rodríguez $L$, Agudelo $C$, Equidad en la política de reforma del sistema de salud. Rev Salud Publica (Bogotá) 2001: 3(1):13-39.

2. Hernández M. Reforma Sanitaria, equidad y derecho a la salud en Colombia. Cad Saude Publica 2002; 18(4):991-1001.

3. Whitehead M. The Concepts and principles of equity and health. World Health Organization. Regional Office for Europe. Copenhague; 1990.

4. Daniels N, Equity of access to health care. Just health Care. Cambridge University Press; 1985.

5. Andersen R, McCutcheon A, Aday LA, Chiu GY, Bell R. Exploring dimensions of access to medical care. Health Serv Res 1983; 18(1): 49-74. 
6. Starfield B. Equity and health: a perspective on nonrandom distribution of health in the population. Rev Panam Salud Publica 2002 Dec;12(6):384-387.

7. Schneider MC, Castillo-Salgado C, Bacallao J, Loyola E, Mujica OJ, Vidaurre M, et al. Métodos de medición de las desigualdades en salud. Rev Panam Salud Publica 2002 Dic; 12(6) 398-414.

8. Alcaldía Mayor de Bogotá, Departamento Administrativo de Planeación Distrital, Centro de Investigaciones para el Desarrollo CID, Universidad Nacional de Colombia. Evolución de los principales indicadores sociales de Bogotá (1990-2003); 2004.

9. Centro de Investigaciones para el Desarrollo CID, Universidad Nacional de Colombia. Pobreza rural: medición y determinantes. Revista del Observatorio de coyuntura socioeconómica No. 16; 2004.

10. Vega R, Hernández A, Barajas C, Conversa L, Cantor B. Evaluación de los efectos de la política de focalización de subsidios en salud y del SISBEN sobre la población vinculada sin capacidad de pago en las localidades de Bosa, Kennedy, Fontibon y Puente Aranda de Bogotá D.C. 2001. Rev Ger Pol Salud 2003; 2(4):72-98.

11. Sen A, ¿Por qué la equidad en salud? Rev Panam Salud Publica 2002 Mayo-Junio; 11(1): 302-309.

12. Castaño R, Arbeláez J, Giedión U, Morales L. Evolución de la Equidad en el Sistema Colombiano de Salud. Naciones Unidas. CEPAL. Unidad de estudios especiales. Secretaría Ejecutiva. Serie: Financiamiento del Desarrollo No 108. Santiago de Chile; 2001.

13. Céspedes J, Jaramillo I, Castaño R. Impacto de la reforma del sistema de seguridad social sobre la equidad en los servicios de salud en Colombia. Cad Saude Publica 2002; 18(4):1003-1024.

14. Organización Panamericana de la Salud, División de Salud y Desarrollo Humano. Proyecto multicéntrico sobre inequidades en el estado de salud, acceso y utilización de los servicios de atención de salud. Washington, D.C.; 2001. 